\section{Anti-CADM-140 Antibody-positive Juvenile Dermatomyositis with Rapidly Progressive Interstitial Lung Disease and Cardiac Involvement}

To the Editor:

Extramusculocutaneous manifestations in juvenile dermatomyositis (JDM) may lead to life-threatening consequences. Interstitial lung disease (ILD) has been reported as one such serious complication in $\mathrm{JDM}^{1,2,3,4}$; however, cardiac involvement in JDM is a rare complication and is seldom report$\mathrm{ed}^{5,6}$. Recently, anti-CADM-140 autoantibody was discovered in amyopathic dermatomyositis and was associated with rapidly progressive ILD $^{7,8}$. We describe a fatal case of JDM complicated by ILD and cardiac involvement in which serum preserved at admission was shown to contain anti-CADM-140 antibody.

A 9-year-old boy was admitted to our hospital with a 4-month history of low-grade fever and erythematous rashes on his face, hands, elbows, and knees. He was developmentally delayed from an unknown cause. He could not describe muscle weakness or tenderness but showed claudication indicating lower-limb muscle weakness. He had Gottron's papules but no heliotrope rash. Cardiac sounds revealed a gallop rhythm and he had fine crackles over both lung fields. His erythrocyte sedimentation rate was 38 $\mathrm{mm} / \mathrm{h}$, white cell count $1800 / \mu 1$, hemoglobin $9.3 \mathrm{~g} / \mathrm{dl}$, platelets $115,000 / \mu 1$, aspartate aminotransferase $207 \mathrm{U} / 1$ (normal 11-39), alanine aminotransferase $101 \mathrm{U} / 1$ (normal 5-40), lactate dehydrogenase $564 \mathrm{U} / 1$ (normal 119-229), aldolase 16.7 U/1 (normal 2.1-6.1), creatine kinase (CK) 104 U/1 (normal 45-160), C-reactive protein (CRP) $0.24 \mathrm{mg} / \mathrm{dl}$ (normal 0.0-0.3), antinuclear antibody 80 (normal < 40), anti-Jo-1 antibody negative, brain natriuretic peptide $55.2 \mathrm{pg} / \mathrm{ml}$ (normal 0.0-19.5), and Krebs von den Lungen-6 (KL-6) $1275 \mathrm{U} / \mathrm{ml}$ (normal 0-500). His electrocardiogram revealed sinus tachycardia at 150 beats/min and $\mathrm{T}$ wave flattening. Echocardiography demonstrated a low ejection fraction of $44 \%$. Chest high-resolution computed tomography (HRCT) revealed bilateral pleural effusions. Whole-body scintigraphy with ${ }^{67} \mathrm{Ga}$-citrate showed increased uptake in the lower lobes of the lungs. Electromyography showed low amplitude and short durations compatible with myopathy. Magnetic resonance imaging showed increased signals at bilateral adductor and pectineal muscles on T2-weighted images. He was diagnosed with JDM and initially treated with oral methylpredonisolone (mPSL). However, his CK increased to $315 \mathrm{U} / 1$ two weeks later and two courses of mPSL pulse therapy were added. After each mPSL pulse therapy ejection fraction was temporarily normalized. One month later the pleural effusions had resolved but new infiltrations developed at the base of both lower lobes on HRCT. We started monthly intravenous cyclophosphamide pulse therapy. Two weeks later, however, fever developed and CRP increased to $4.60 \mathrm{mg} / \mathrm{dl}$. Bacterial cultures from blood, sputum, and aspirated pleural effusion were negative. Serum B-D-glucan and cytomegalovirus antigen were also negative. Pneumocystis jiroveci was not detected in aspirated pleural effusion. Repeat HRCT demonstrated progressive bilateral infiltration in the lower lobes, then intravenous cyclosporin A was administered. KL-6 was elevated to $5138 \mathrm{U} / \mathrm{ml}$. His respiratory condition rapidly deteriorated and pneumomediastinum, subcutaneous emphysema, and left pneumothorax developed (Figure 1). He was intubated and mechanically ventilated and underwent plasmapheresis. Despite intensive treatment, he died 1 week after intubation, which was 3 months after the initial admission to hospital.

With his guardian's consent, an autopsy was performed. The lungs were entirely firm and showed remarkable congestion, and the microscopic examination revealed intense alveolar hemorrhage, formation of hyaline membrane, and infiltration of inflammatory cells suggesting diffuse alveolar damage (Figure 2). Neither interstitial changes nor infection of the lungs were present. The heart demonstrated hypertrophy and histologic examination detected no specific changes of myocarditis, pericarditis, endocarditis, or fibrosis except for partial degeneration of the papillary muscles of the left ventricle. Serum anti-CADM-140 antibody was measured by ELISA in his serum preserved at admission (courtesy of Dr. Masataka Kuwana, Keio University of Japan) ${ }^{7,8}$. Anti-CADM-140 antibody was elevated (25.256 units, normal below 8 ).
ILD is a rare but serious complication of $\mathrm{JDM}^{1,2,3,4}$. In adult cases of polymyositis (PM) and dermatomyositis (DM), histologic findings of ILD have been shown to be correlated with clinical outcomes ${ }^{9,10}$. In particular, cases of PM/DM with diffuse alveolar damage have shown the worst prognosis among those with major histopathology of ILD, as observed in our case $^{9,10}$. Our patient had impaired cardiac function during his admission. At autopsy, partial degeneration of the papillary muscles of the left ventricle was the only finding. It is unclear whether this histologic change is sufficient to explain his impaired cardiac function. In previous reports, the cardiac features seen most often in cases of PM/DM/JDM were myocarditis, myocardial fibrosis, and vascular abnormalities on histologic examination, features not seen in our case $\mathrm{e}^{5,6}$.

Anti-CADM-140 antibody was recently discovered in clinically amyopathic patients with DM and its presence was associated with rapidly progressive $\operatorname{ILD}^{7,8}$. The presence of anti-CADM-140 antibody in our patient may suggest that this antibody is a predictor of rapidly progressive ILD and poor prognosis even in JDM.

We describe a case of fatal JDM with rapidly progressive ILD and cardiac involvement. Positive anti-CADM-140 antibody may predict a poor prognosis for JDM with ILD.

NODOKA SAKURAI, MD; KAZUSHIGE NAGAI, MD, PhD; HIROYUKI TSUTSUMI, MD, PhD, Department of Pediatrics; SHINGO ICHIMIYA, MD, PhD, Department of Pathology, Sapporo Medical University School of Medicine, South 1, West 16, Chuo-ku, Sapporo, Hokkaido 060-8543, Japan. Address correspondence to Dr. Nagai; E-mail: nkazu@sapmed.ac.jp

\section{REFERENCES}

1. Lin Y-C, Yang Y-H, Lin Y-T, Chiang B-L. Steroid refractory interstitial pneumonitis in a patient with juvenile dermatomyositis. J Microbiol Immunol Infect 2002;35:259-61.

2. Kobayashi I, Yamada M, Takahashi Y, Kawamura N, Okano M, Sakiyama Y, et al. Interstitial lung disease associated with juvenile dermatomyositis: clinical features and efficacy of cyclosporin A. Rheumatology 2003;43:371-4.

3. Tosun A, Serdaroglu G, Aslan MT, Polat M, Akalm T, Tekgul H et al. Severe juvenile dermatomyositis: two patients complicated with extra musculocutaneous involvement. Rheumatol Int 2006;26:1040-3.

4. Zulian F, Martinez Toledo MM, Amigoni A, Martini G, Agosto C, Pettenazzo A. Successful use of extracorporeal membrane oxygenation for severe interstitial lung disease in a child with dermatomyositis. Intensive Care Med 2007;33:1663-6.

5. Denbow CE, Lie JT, Tancredi RG, Bunch TW. Cardiac involvement in polymyositis. A clinicopathologic study of 20 autopsied patients. Arthritis Rheum 1979;22:1088-92.

6. Haupt HM, Hutchins GM. The heart and cardiac conduction system in polymyositis-dermatomyositis: a clinicopathologic study of 16 autopsied patients. Am J Cardiol 1982;50:998-1006.

7. Sato S, Hirakata M, Kuwana M, Suwa A, Inada S, Mimori T et al. Autoantibodies to a 140-kd polypeptide, CADM-140, in Japanese patients with clinically amyopathic dermatomyositis. Arthritis Rheum 2005;52:1571-6.

8. Sato S, Hoshino K, Satoh T, Fujita T, Kawakami Y, Fujita T, et al. RNA helicase encoded by melanoma differentiation-association gene 5 is a major autoantigen in patients with clinically amyopathic dermatomyositis. Arthritis Rheum 2009;60:2193-200.

9. Tazelaar HD, Viggiano RW, Pickersgill J, Colby TV. Interstitial lung disease in polymyositis and dermatomyositis. Clinical features and prognosis as correlated with histologic findings. Am Rev Respir Dis 1990;141:727-33.

10. Fathi M, Lundberg I. Interstitial lung disease in polymyositis and dermatomyositis. Curr Opin Rheumatol 2005;17:701-6.

J Rheumatol 2011;38:5; doi:10.3899/jrheum.101220 


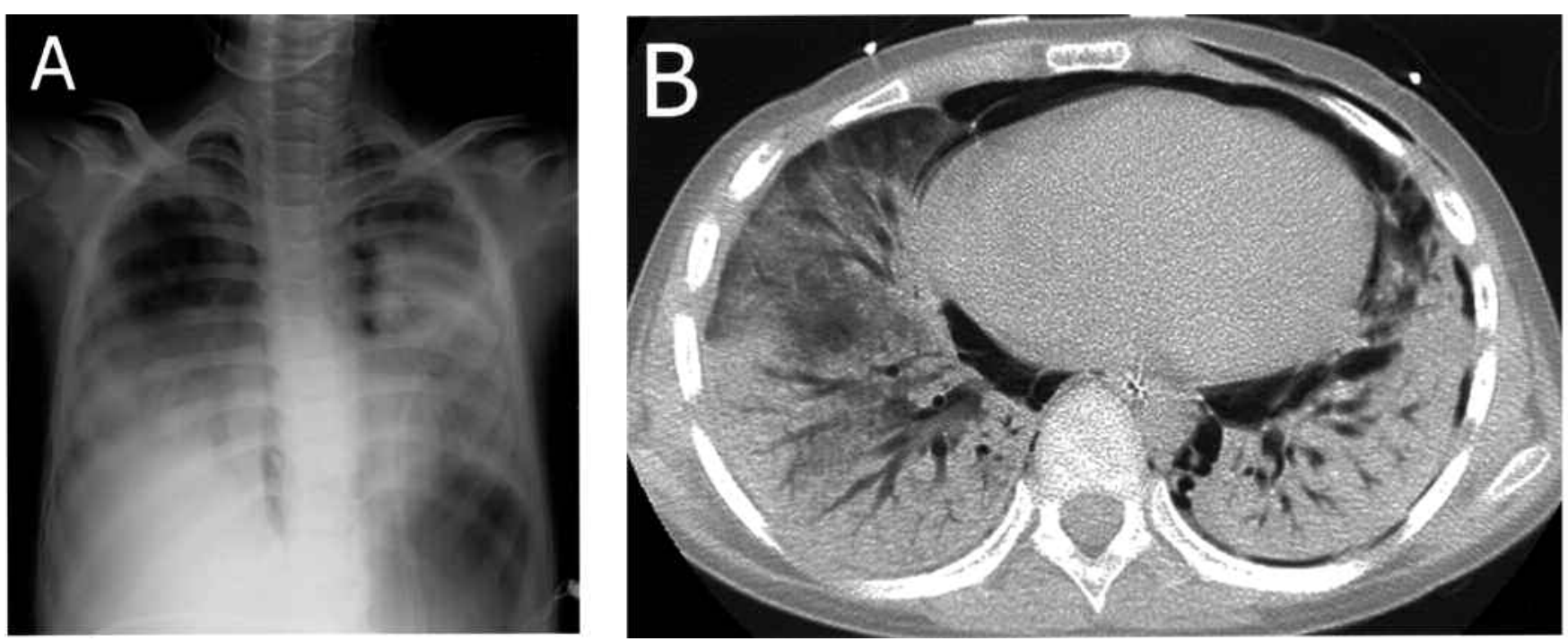

Figure 1. A. Chest radiography showing diffuse bilateral consolidation of the lungs with pneumomediastinum and subcutaneous emphysema of thoracic walls. B. Chest HRCT shows extensive bilateral lobar consolidation, pneumomediastinum, subcutaneous emphysema of the thoracic walls, and left pneumothorax.
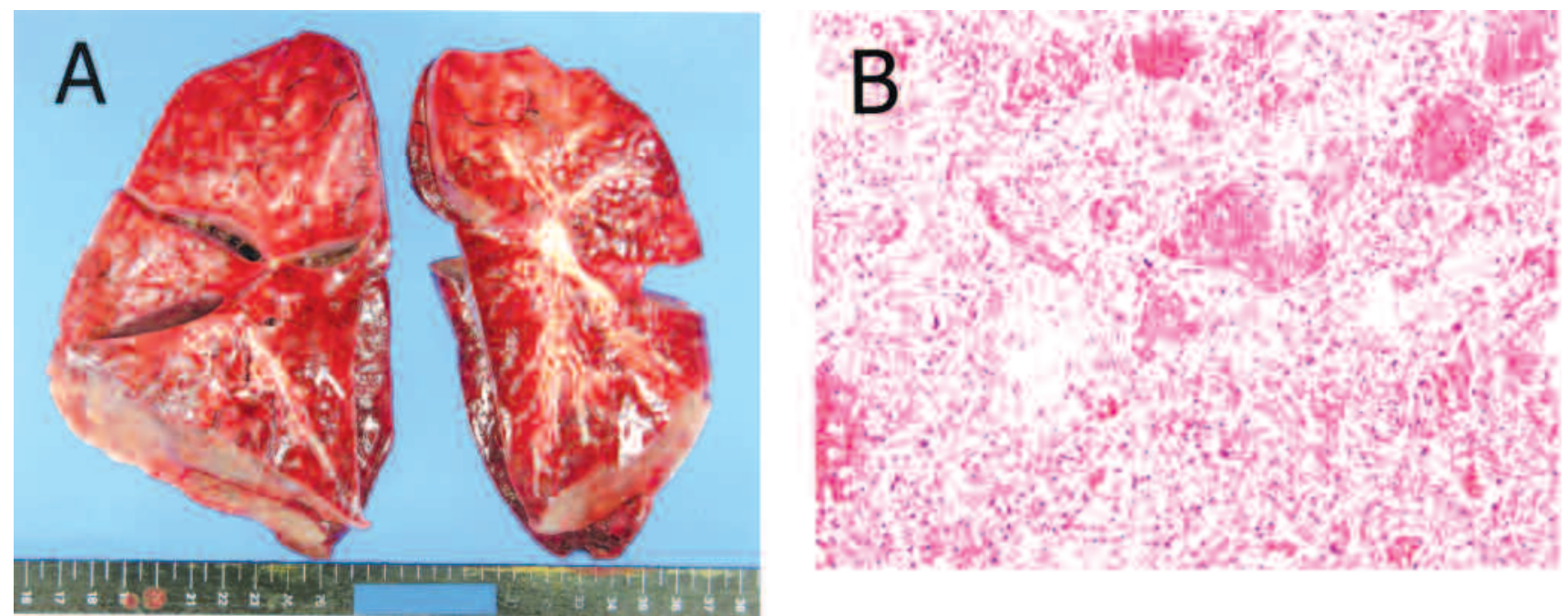

Figure 2. Autopsy findings of the lungs. A. Severe congestion of the lungs. B. The lung histology shows alveolar hemorrhage, formation of hyaline membrane, and infiltration of inflammatory cells compatible with diffuse alveolar damage (H\&E stain, original magnification $\times 200)$. 